\title{
Rare and Hadronic B decays with CLEO
}

\author{
Eckhard von Toerne* for the CLEO Collaboration \\ Address: The Ohio State University, Columbus OH 43210 \\ E-mail: 'evt@mps.ohio-state.edu'
}

Abstract: Based on the CLEO II and II.5 data sets CLEO has observed several new rare and hadronic $\mathrm{B}$ decays and also updated the $\mathrm{b} \rightarrow \mathrm{s} \gamma$ measurement.

Rare and hadronic B meson decays provide an excellent experimental field to test the Standard Model of particle physics. While effects like CP-violation have been observed in hadronic B decays $B \rightarrow \mathrm{J} / \Psi \mathrm{K}_{\mathrm{s}}^{0}$, the extraction of CKM-matrix elements and phases might still prove difficult since many decay channels have to be measured and effects like final state interactions, re-scattering and interference between dominant and suppressed decay

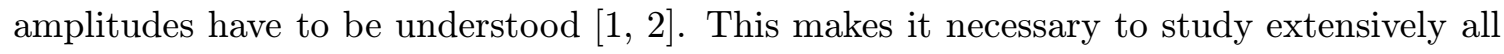
rare and hadronic B decays to gain full understanding of the dynamics in B decays.

The results presented here are based on the CLEO II and II.5 data sets. The CLEO detector is located at the CESR $\mathrm{e}^{+} \mathrm{e}^{-}$collider in Ithaca, NY. An integrated luminosity of $9.1 \mathrm{fb}^{-1}$ was collected on the $\Upsilon(4 \mathrm{~S})$ resonance and $4.3 \mathrm{fb}^{-1} \sim 60 \mathrm{MeV}$ below the resonance to study the continuum background from $\mathrm{e}^{+} \mathrm{e}^{-} \rightarrow \mathrm{q} \overline{\mathrm{q}}$. The kinematics of the $\Upsilon(4 \mathrm{~S})$ decay, in which two B mesons with equal masses are produced, allow us to define two sensitive variables: the Beam-constrained mass $\mathrm{M}_{\mathrm{B}}=\sqrt{\mathrm{E}_{\text {beam }}^{2}-\mathrm{P}_{\mathrm{B}}^{2}}$ and the Energy difference $\Delta \mathrm{E}=\mathrm{E}_{\mathrm{B}}-\mathrm{E}_{\text {beam }}$, where $\mathrm{E}_{\mathrm{B}}$ and $\mathrm{P}_{\mathrm{B}}$ are the measured energy and momentum of the $\mathrm{B}$ candidate and $\mathrm{E}_{\mathrm{beam}}$ is the beam energy.

1. $\mathrm{b} \rightarrow \mathrm{s} \gamma$

Many of the rare B decay modes can be enhanced by non-Standard Model contributions. The most prominent example might be $b \rightarrow s \gamma$. This flavor changing neutral current does not occur as a first-order Standard Model process but is allowed via penguin diagrams. Non-Standard Model decays, where the $\mathrm{W}^{-}$in the penguin loop is replaced by a charged Higgs or Chargino, would alter the branching fraction.

The $\mathrm{b} \rightarrow \mathrm{s} \gamma$ measurement presented here has increased statistical significance and updates a previous CLEO result [i3] reduced by extending the signal window from $\mathrm{E}_{\gamma}=2.2-2.7 \mathrm{GeV}$ to $2.0-2.7 \mathrm{GeV}$.

${ }^{*}$ Speaker. 


\subsection{Extraction of the $b \rightarrow s \gamma$ signal}

Even considering the inclusive sample, $\mathrm{b} \rightarrow \mathrm{s} \gamma$ is a rare decay. $\pi^{0}$ or $\eta$ mesons produce a large background of real photons in addition to events with ISR. Fig. 1'i-(a) demonstrates the signal and background levels on a logarithmic scale. The dominant background comes from continuum events. $B \bar{B}$ events contribute with a much softer photon spectrum.

Continuum ISR events are excluded by a cut on the polar angle $\left|\cos \vartheta_{\gamma}\right|<0$.7. Photons are vetoed if they can be combined with other photons to form a $\pi^{0}$ or $\eta$. Continuum background is further suppressed by collecting event information that can separate the continuum from $\mathrm{B} \overline{\mathrm{B}}$, using event shape variables and pseudo-reconstruction of hadronic $\mathrm{B}$ decays. In the first pseudo-reconstruction method, $\mathrm{B}$ candidates are formed from the photon candidate and additional kaon and pion tracks. The second method uses identified leptons to tag semi-leptonic decays of the companion B. Both methods allow us to measure the flavor of the B, which will be used in the CP-asymmetry measurement.

All the candidate's quantities are combined into one variable using a neural net. The neural net output is used as a weight, which peaks at +1 for $b \rightarrow s \gamma$ events. The weighted energy spectra for on- and off-resonance data (scaled to luminosity) are subtracted. The weighting increases the statistical significance of the on-resonance signal. The $\mathrm{B} \overline{\mathrm{B}}$ background is subtracted in two steps. First we subtract the measured spectra of photons from $\pi^{0}$ and $\eta$ decay. The remaining background has contributions from $\mathrm{B}$ decays with successive electro-magnetic decays like radiative $J / \Psi$ or $\omega$ decays. Only the tail of the photon energy spectrum reaches the signal region. The remaining background is estimated with a detailed Monte Carlo simulation and is also subtracted. The photon energy spectrum after subtraction is shown in Fig. 笽-b. A signal peak is prominent at $E_{\gamma} \approx 1 / 2 \mathrm{M}_{\mathrm{B}}$. The sidebands of the peak are consistent with zero. The preliminary branching fraction

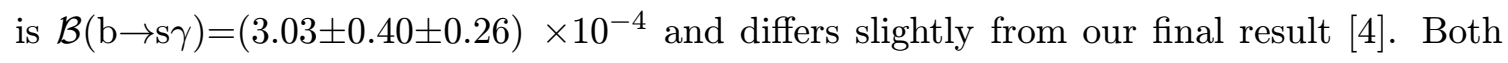

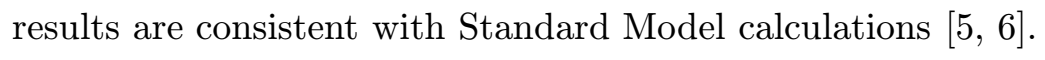

The $\mathrm{E}_{\gamma}$ spectrum is fitted using a parameterization of the spectator model [i, $]$ ] with the b-quark mass and the fermi momentum of the $b$ as free parameters. The fit results are also shown in Fig. iilib. The calculation of these parameters can be utilized to reduce the uncertainty of $\mathrm{V}_{\mathrm{cb}}$ and $\mathrm{V}_{\mathrm{ub}}$ measurements in semi-leptonic $\mathrm{B}$ decays [i]

The CP-asymmetry in $\mathrm{b} \rightarrow \mathrm{s} \gamma$ is defined as $\mathcal{A}_{\mathrm{CP}}=\left(\Gamma\left(\mathrm{N}_{\mathrm{b}}\right)-\Gamma\left(\mathrm{N}_{\overline{\mathrm{b}}}\right)\right) /\left(\Gamma\left(\mathrm{N}_{\mathrm{b}}\right)+\Gamma\left(\mathrm{N}_{\overline{\mathrm{b}}}\right)\right)$. We find no significant asymmetry: $\mathcal{A}_{\mathrm{CP}}=(-0.079 \pm 0.108 \pm 0.022)(1.0 \pm 0.030)$ [9.9]. The first error is statistical, the second is the additive systematic error, and the multiplicative systematic error comes from the systematic uncertainties on the tagging efficiency and background subtraction. We also derive $90 \%$ C.L. limits on $\mathcal{A}_{\mathrm{CP}}:-0.27<\mathrm{A}_{C P}<+0.10$.

\section{Searches for $\mathrm{B} \rightarrow \mathrm{K}^{(*)} \ell^{+} \ell^{-}$and $\mathrm{B}^{+} \rightarrow \mathrm{D}^{*+} \mathrm{K}_{\mathrm{s}}^{0}$}

We searched for $\mathrm{B} \rightarrow \mathrm{K}^{(*)} \ell^{+} \ell^{-}$, where $\ell$ is either an electron or a muon. In decays with a $\mathrm{K}^{*}$ we impose a cut on the $\ell^{+} \ell^{-}$invariant mass of $m_{\ell^{+} \ell^{-}}>0.5 \mathrm{GeV}$ to remove the contributions from the virtual photon pole. We derive upper limits of $\mathcal{B}\left(\mathrm{B} \rightarrow \mathrm{K} \ell^{+} \ell^{-}\right)<$ $1.7 \times 10^{-6}, \mathcal{B}\left(\mathrm{B} \rightarrow \mathrm{K}_{(892)}^{*} \ell^{+} \ell_{m_{\ell \ell}>0.5 \mathrm{GeV}}^{-}\right)<3.3 \times 10^{-6}\left[{ }_{L}^{1} \hat{O}_{1}^{1}\right]$. We obtain a combined limit of 

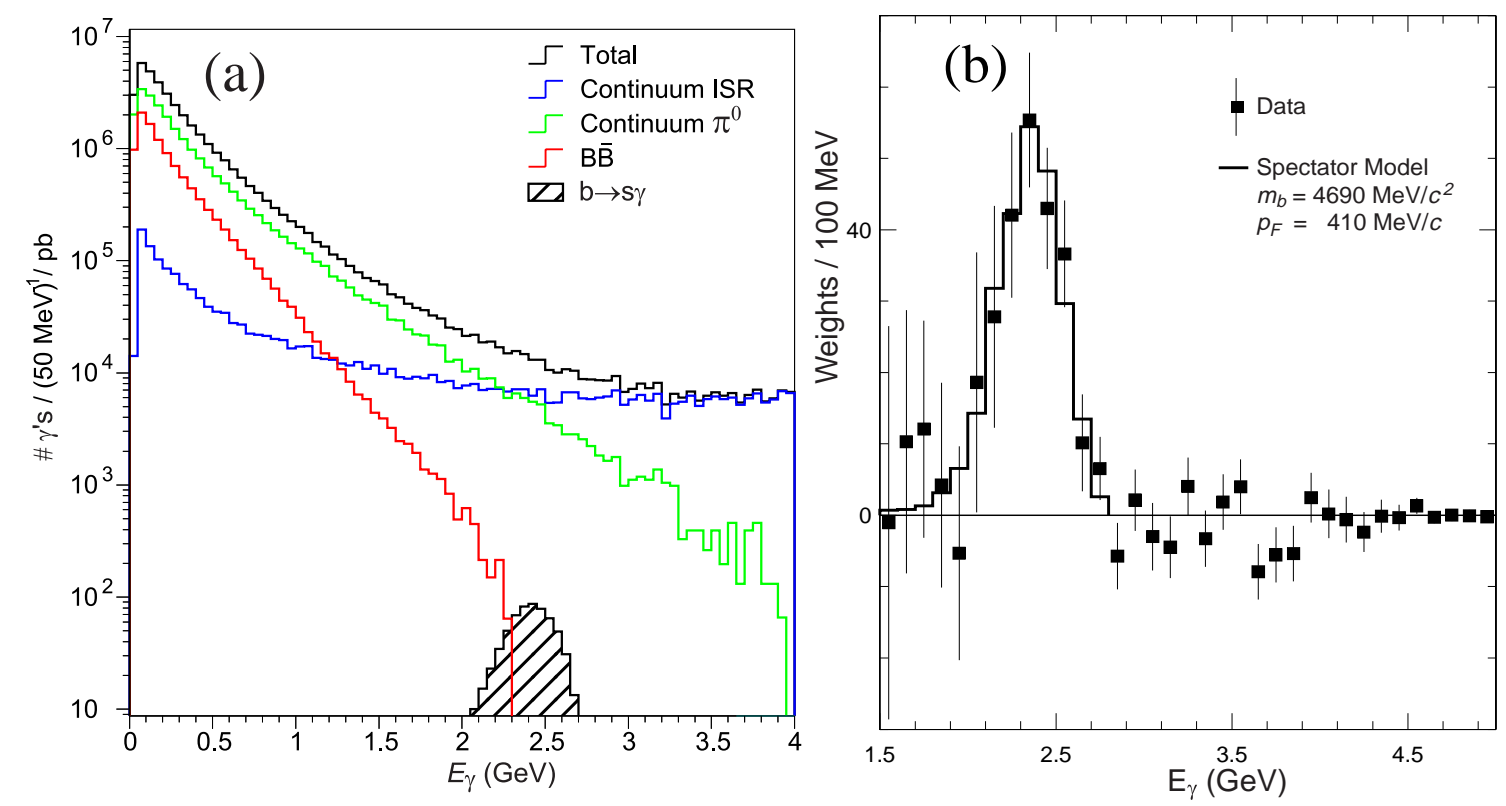

Figure 1: (a) Signal and background levels for $\mathrm{b} \rightarrow \mathrm{s} \gamma$. (b) Photon energy spectrum after background subtraction; a clear signal peak is observed at about half the B mass. The spectator model fit is shown as the histogram.

$\mathcal{B}\left(\mathrm{B} \rightarrow \mathrm{K}^{(*)} \ell^{+} \ell^{-}\right)<1.5 \times 10^{-6}$ at $90 \%$ C.L., which is only $50 \%$ higher than the Standard

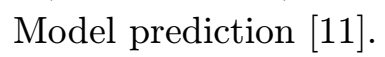

CLEO has also searched for the decay $\left.\mathrm{B}^{+} \rightarrow \mathrm{D}^{*+} \mathrm{K}_{\mathrm{s}}^{0}[1] \overline{1} 2 \overline{2}\right]$. This decay proceeds through an annihilation diagram that is proportional to $\mathrm{V}_{\mathrm{ub}}$. We observe zero candidates while expecting $0.29 \pm 0.05$ background events and derive an upper limit of $\mathcal{B}\left(\mathrm{B}^{+} \rightarrow \mathrm{D}^{*+} \mathrm{K}_{\mathrm{s}}^{0}\right)<$ $9.5 \times 10^{-5}$ at $90 \%$ C.L.

\section{Factorization tests in $\mathrm{B} \rightarrow \mathrm{D}^{(*)} 4 \pi$}

In a recent publication [13 3 in CLEO has studied the decay $\mathrm{B} \rightarrow \mathrm{D}^{(*)} \pi^{-} \pi^{-} \pi^{+} \pi^{0}$. The fourpion mass spectrum can be compared to the spectrum in tau decays $\tau^{-} \rightarrow \pi^{-} \pi^{-} \pi^{+} \pi^{0} \nu$.

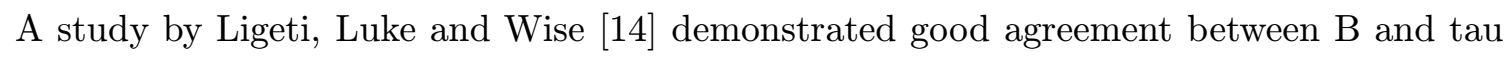
decay data over the accessible mass range of $0.6-1.7 \mathrm{GeV}$. Their comparison tested the factorization hypothesis as a function of the four-pion mass. The validity of this test might be limited because of additional contributions ${ }^{1}$ to $\mathrm{B} \rightarrow \mathrm{D}^{*}(4 \pi)^{-}$, that are not possible in tau decays. These additional contributions can be measured in a related decay mode $\overline{\mathrm{B}}^{0} \rightarrow \mathrm{D}^{* 0} \pi^{+} \pi^{+} \pi^{-} \pi^{-}$. This mode is suppressed since the net charge of the pions is zero and the pions cannot come from the $\mathrm{W}^{-}$decay alone. CLEO has observed this decay for the first time with a branching fraction of $\mathcal{B}\left(\overline{\mathrm{B}}^{0} \rightarrow \mathrm{D}^{* 0} \pi^{+} \pi^{+} \pi^{-} \pi^{-}\right)=(0.30 \pm 0.07 \pm 0.06) \%\left[1 \overline{1} \overline{5}_{1}^{1}\right]$. In the four-pion mass range $0.6-1.7 \mathrm{GeV}$, the $\overline{\mathrm{B}}^{0} \rightarrow \mathrm{D}^{* 0} \pi^{+} \pi^{+} \pi^{-} \pi^{-}$contribution is consistent with zero. This supports the validity of the factorization test by Ligeti et al.

\footnotetext{
${ }^{1}$ E.g. $\mathrm{B} \rightarrow(4 \pi)^{-}$decays in which not all pions come from the virtual $\mathrm{W}^{-}$.
} 


\section{Color-suppressed decays $\overline{\mathrm{B}}^{0} \rightarrow \mathrm{D}^{(*) 0} \pi^{0}$}

The decay $\overline{\mathrm{B}}^{0} \rightarrow \mathrm{D}^{(*) 0} \pi^{0}$ proceeds predominantly through the internal spectator diagram. This decay mode is color-suppressed, since the color of the quark-pair originating from the W decay must match the color of the other quark pair. The observation of $\overline{\mathrm{B}}^{0} \rightarrow \mathrm{D}^{(*) 0} \pi^{0}$ completes the measurement of $\mathrm{D}^{(*)} \pi$ final states and allows us to extract the strong phase

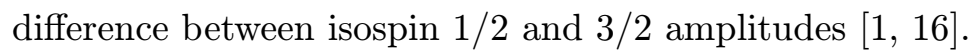

$\mathrm{B}$ mesons are reconstructed by selecting high-momentum $\mathrm{D}^{(*) 0}$ and $\pi^{0}$ mesons. The kinematic resolution of $\pi^{0}$ and $\mathrm{D}^{(*) 0}$ candidates is improved by mass-constrained kinematic fits. We accept $\mathrm{B}$ candidates with $\mathrm{M}_{\mathrm{B}}$ above $5.24 \mathrm{GeV}$ and $|\Delta \mathrm{E}|<300 \mathrm{MeV}$. For each candidate we calculate the sphericity vectors of the $\mathrm{B}$ daughter particles and of the rest of the event. We require the cosine of the angle between these two vectors to be within -0.8 and 0.8 .

The signal yield is obtained from an unbinned, extended maximum likelihood fit. The free parameters of the fit are the number of signal events, background from B decays, and from continuum $\mathrm{e}^{+} \mathrm{e}^{-}$annihilation. Four variables are used as input to the maximum likelihood fit: the beam-constrained mass $\mathrm{M}_{\mathrm{B}}$, the energy difference $\Delta \mathrm{E}$, the Fisher Discriminant $\mathcal{F}_{\mathrm{D}}$, which is a combination of event shape variables, and the cosine of the decay angle of the $\mathrm{B} \cos \theta_{\mathrm{BHel}}$, defined as the angle between the $\mathrm{D}^{(*)}$ momentum and the $\mathrm{B}$ flight direction calculated in the $\mathrm{B}$ rest frame. The likelihood of the $\mathrm{B}$ candidate is the sum of probabilities for the signal and two background hypotheses with relative weights maximizing the likelihood. Monte Carlo experiments are performed to test the fitting procedure and to obtain the relation between fit yield and signal branching fractions.

We observe both $\overline{\mathrm{B}}^{0} \rightarrow \mathrm{D}^{(*) 0} \pi^{0}$ decay modes with preliminary branching fractions: $\mathcal{B}\left(\overline{\mathrm{B}}^{0} \rightarrow \mathrm{D}^{0} \pi^{0}\right)=(2.6 \pm 0.3 \pm 0.6) \times 10^{-4}$ and $\mathcal{B}\left(\overline{\mathrm{B}}^{0} \rightarrow \mathrm{D}^{* 0} \pi^{0}\right)=(2.0 \pm 0.5 \pm 0.7) \times 10^{-4}$, which differ slightly from our final results [1] $\left.{ }_{1}^{1} \overline{1}\right]$. Fig. $\overline{2}_{1}$ demonstrates the significance of our results which is above 4 sigma for each decay mode. Our measurements allow us to calculate the strong phase difference between isospin $1 / 2$ and $3 / 2$ amplitudes. We obtain $\cos \delta_{I, D}=0.90 \pm 0.09$ and $\cos \delta_{I, D *}=0.91 \pm 0.08$, for $\mathrm{D} \pi$ and $\mathrm{D}^{*} \pi$, respectively. We quote the cosine of the angle because the errors on the cosine are gaussian to a good approximation. The error distribution of the angle $\delta_{I}$ is highly asymmetric and non-gaussian. A detailed discussion can be found in a publication by Neubert and Petrov [i] preliminary results and preliminary results obtained by the BELLE Collaboration [i] $\left.{ }_{1}^{1} \bar{g}\right]$.

\section{References}

[1] M. Neubert and B. Stech in Heavy Flavors, edited by A.J. Buras and M. Lindner (World Scientific, Singapore, 2nd edition 1998).

[2] T. E. Browder, K. Honscheid and D. Pedrini, Ann. Rev. Nucl. Part. Sci. 46, 395 (1996).

[3] CLEO Collaboration, M. S. Alam et al., Phys. Rev. Lett. 742885 (1995).

[4] Final result: $\mathcal{B}(\mathrm{b} \rightarrow \mathrm{s} \gamma)=\left(3.21 \pm 0.43 \pm 0.27_{-0.10}^{+0.18}\right) \times 10^{-4}$ CLEO Collaboration, S. Chen et al., hep-ex/0108032, subm. to Phys. Rev. Lett.

[5] K. Chetyrkin, M. Misiak, and M. Münz, Phys. Lett. B400206 (1997). 

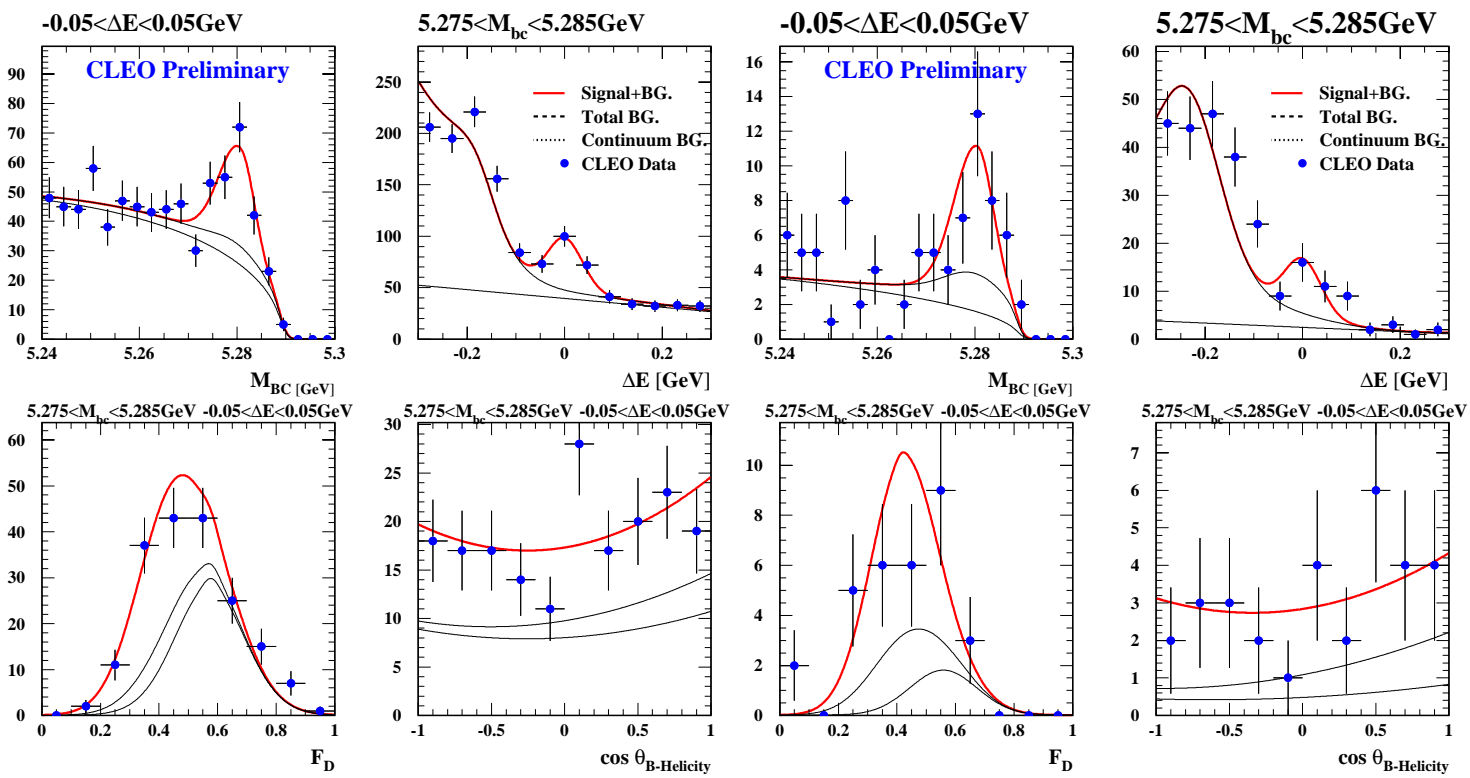

Figure 2: Fit input variable distributions. Left side $\overline{\mathrm{B}}^{0} \rightarrow \mathrm{D}^{0} \pi^{0}$, right side $\overline{\mathrm{B}}^{0} \rightarrow \mathrm{D}^{* 0} \pi^{0}$. The results of the unbinned, extended maximum likelihood fit are shown as the full line. The dotted line represents the fitted continuum and the dashed line is the fit result for the sum of $\mathrm{B} \overline{\mathrm{B}}$ and continuum background. To enhance the signal for display purposes, the fit results are projected into the $\mathrm{M}_{\mathrm{B}}-\Delta \mathrm{E}$ signal region $-0.05<\Delta \mathrm{E}<0.05 \mathrm{GeV}, 5.275<\mathrm{M}_{\mathrm{B}}<5.285 \mathrm{GeV}$.

[6] P. Gambino and M. Misiak, hep-ph/0104034.

[7] A. Ali and C. Greub, Phys. Lett. B259 182 (1991).

[8] D. Miller, same proceedings; and D. Cronin-Hennessy et al. (CLEO), hep-ex/0108033.

[9] CLEO Collaboration, T. E. Coan et al., Phys. Rev. Lett. 865661 (2001).

[10] CLEO Collaboration, S. Anderson et al., Phys. Rev. Lett. 87181803 (2001).

[11] A. Ali et al., Phys. Rev. D 61074024 (2000).

[12] CLEO Collaboration, A. Gritsan et al., Phys. Rev. D 64 077501, (2001).

[13] CLEO Collaboration, J. P. Alexander et al., Phys. Rev. D 64092001 (2001).

[14] Z. Ligeti, M. Luke, and M. B. Wise, Phys. Lett. B507 142 (2001).

[15] CLEO Collaboration, K. W. Edwards et al., hep-ex/0105071, subm. to Phys. Rev. Lett.

[16] J.L. Rosner, Phys. Rev. D 60, 074029 (1999).

[17] CLEO Collaboration, T. E. Coan et al., hep-ex/0110055, subm. to Phys. Rev. Lett. Final Results: $\mathcal{B}\left(\overline{\mathrm{B}}^{0} \rightarrow \mathrm{D}^{0} \pi^{0}\right)=\left(2.74_{-0.32}^{+0.36} \pm 0.55\right) \times 10^{-4}, \mathcal{B}\left(\overline{\mathrm{B}}^{0} \rightarrow \mathrm{D}^{* 0} \pi^{0}\right)=\left(2.20_{-0.52}^{+0.59} \pm 0.79\right) \times 10^{-4}$

[18] M. Neubert and A. Petrov, Phys. Lett. B519, 50 (2001).

[19] R-S. Lu (same proceedings) and hep-ex/0107048; Final results by the BELLE Collaboration: hep-ex/0109021, subm. to Phys. Rev. Lett. 